\title{
In response to Li et al.: Linker histones function in Drosophila embryogenesis
}

Albert Carbonell1,2,", Lazslo Henn ${ }^{3,{ }^{*}}$, Juan Pérez-Roldán ${ }^{1,2}$, Srividya Tamirisa ${ }^{1,2}$, Anikó Szabó ${ }^{3,5}$, Imre M. Boros ${ }^{3,4}$ and Fernando Azorín ${ }^{1,2,+}$

${ }^{1}$ Institute of Molecular Biology of Barcelona, IBMB, CSIC. Baldiri Reixac, 4. 08028 Barcelona. Spain.

${ }^{2}$ Institute for Research in Biomedicine, IRB Barcelona. The Barcelona Institute of Science and Technology. Baldiri Reixac, 10. 08028 Barcelona. Spain

IInstitute of Biochemistry, Biological Research Centre of Szeged, Szeged, H6726, Hungary

${ }^{4}$ Department of Biochemistry and Molecular Biology, Faculty of Science and Informatics, University of Szeged, Szeged, H-6726, Hungary

${ }^{5}$ Doctoral School in Biology, Faculty of Science and Informatics, University of Szeged, Szeged, H-6726, Hungary

Key words: linker histone $\mathrm{H} 1 /$ dBigH1/ chromatin/ embryogenesis/ ZGA/ Drosophila

${ }^{*}$ Equal contribution

+Corresponding author:

Dr. F. Azorín

Institute of Molecular Biology of Barcelona, CSIC

Institute for Research in Biomedicine, IRB Barcelona

Baldiri Reixac, 10. 08028 Barcelona. Spain

Phone: 3493-4034958

Fax: 3493-4034979

e-mail: fambmc@ibmb.csic.es 


\section{ABSTRACT}

In an earlier paper (Pérez-Montero et al., 2013), we reported that the embryonic linker histone of Drosophila dBigH1 was essential for early Drosophila embryogenesis since embryos homozygous for the bigH1100 mutation showed strong defects and did not survive beyond zygotic genome activation (ZGA) at cellularization. Recent results challenge these observations since null bigH1 mutations generated by CRISPR/Cas9 methodology turn out to be homozygous viable, as reported in Li et al. (2019) and here. In this regard, Li et al. described a novel mechanism by which lack of $\mathrm{dBigH} 1$ is compensated by the early expression of maternal $\mathrm{dH} 1$. Here, we confirm this observation and show that such compensatory mechanism is not activated in bigH1 100 embryos.

\section{INTRODUCTION}

Metazoans usually encode multiple histone $\mathrm{H} 1$ variants, some of which are specifically expressed in the germline (reviewed in Pérez-Montero et al., 2016). In vertebrates, female and male germline specific $\mathrm{H} 1 \mathrm{~s}$ are usually different. Female-specific variants accumulate in the oocyte and are retained during early embryogenesis (Pérez-Montero et al., 2016). In Drosophila, H1 complexity is low since it encodes for a single ubiquitously expressed somatic $\mathrm{dH} 1$ variant and a single germline specific dBigH1 variant (reviewed in Bayona-Feliu et al., 2016). dBigH1 is expressed in both the female and male germline, and it is present in the early embryo until zygotic genome activation (ZGA) at cellularization (Carbonell et al., 2017; Pérez-Montero et al., 2013). At this stage, $\mathrm{dBigH} 1$ is replaced by somatic $\mathrm{dH} 1$ in somatic cells, whereas it is retained in the primordial germ cells (PGC) (Pérez-Montero et al., 2013). In previous studies, we generated a loss-of-function bigH1 ${ }^{100}$ allele that showed strong embryonic lethality with no homozygous bigH1100 embryos surviving cellularization (PérezMontero et al., 2013), suggesting that $\mathrm{dBigH} 1$ is essential for early embryo development. Recent results by Li et al. challenged this view since null bigH1 CRISPR alleles were found to be homozygous viable (Li et al., 2019). Notably, 
$\mathrm{Li}$ et al. showed that the lack of $\mathrm{dBigH} 1$ was compensated by the early expression of maternal $\mathrm{dH} 1$ (Li et al., 2019).

\section{MATERIALS AND METHODS}

\section{Drosophila stocks}

bigH1100 allele is described in (Pérez-Montero et al., 2016). bigH1NULL and bigH1NSTOP alleles were generated by CRISPR/Cas9 mediated homologous $\begin{array}{llll}\text { recombination. } & \text { CRISPR } & \text { Optimal } & \text { Target }\end{array}$ (http://targetfinder.flycrispr.neuro.brown.edu/) was used for identification of gRNA target sites downstream and upstream of the $d B i g H 1$ encoding genomic locus. Upstream (5'-ATTAGCAGTGTTATTCCATA-3') and downstream (5'ATAATACCTCTAGAAGGAAT-3') gRNA sequences were cloned into pCFD4 plasmid (Port et al., 2014) and injected into $y[1]$ v[1] P\{nos-phiC31lint.NLS\}X; $P\{$ CaryP\}attP40 embryos (Bischof et al., 2007) (500ng/ $\mu$ l plasmid DNA in injection buffer $\left.\left(5 \mathrm{mM} \mathrm{KCl}, 0.1 \mathrm{mM} \mathrm{KH}_{2} \mathrm{PO}_{4}, \mathrm{pH}: 7.8\right)\right)$ to insert transgenic gRNA genes into the 2nd chromosome of the Drosophila genome.

A 4640 bp long genomic fragment containing the $d B i g H 1$ locus was amplified with dBigH1 Rev (5'-GGACACACTGACATTTAGCTGTTTGG-3') and dBigH1 Fw (5'-TACTCCGTAATTGATGAGATTCCGCC-3') primers and inserted into $\mathrm{pTZ57R/T}$ plasmid (Thermofischer Scientific). On this donor plasmid PAM sequences of upstream and downstream gRNA target sites were mutated by PCR mutagenesis using BigH1 PAM upstream (5'ACCTTCCGTACGCTCATTTTTAGAATTAACTCCTCTTCTTCTTTAGCAGTGT TAACATATG-3') and BigH1 PAM downstream (5'AACTCAATTGAAATGAGAAAATGTGTTTATAATAGCTCTAGAAGGAATAAGC GATAACCGAG-3') primers. bigH1NSTOP sequence was generated by PCR mutagenesis using NSTOP primer (5'-CGATTCTGACAACCCCAAGTCGATGG TCTGAAAACCAAAGGG-3') exchanging P58 with STOP codon and 3xFLAG epitope encoding sequence was built following the first methionine. $3 \times \mathrm{P} 3$ promoter driven dsRed sequence as a marker gene was introduced into bigH1NSTOP allele carrying donor plasmid, followed by $16 \mathrm{bp}$ of the $\mathrm{dBigH1}$ 5'UTR. bigH1NULL sequence was generated by Sequence and Ligation 
Independent Cloning (SLIC) (Jeong et al., 2012). In this case the entire dBigH1 coding sequence was replaced with mCherry sequence surrounded by loxP sites. For SLIC reactions the following primers were used: loxP 5UTR (5'CTTCGTATAATGTATGCTATACGAAGTTATCATGTTATTAGTTGGAAATTAA ATTGAACAAAAATTAGAAATACAACTC-3'), loxP mCh Fw (5'-TCGTATAGCAT ACATTATACGAAGTTATTAGTGAGCAAGGGCGAGGAGGA-3'), IoxP उUTR (5'-TTCGTATAGCATACATTATACGAAGTTATAATTATGATTTATATGTTTTTT TTTCAGTACATGTG-3'), and loxP mCh Rev (5'-ACTTCGTATAATGTATGCTA TACGAAGTTATCTTGTACAGCTCGTCCATGCCG-3'). bigH1NSTOP and bigH1NULL encoding donor plasmids were injected $(500 \mathrm{ng} / \mu \mathrm{l}$ plasmid DNA in injection buffer) into embryos laid by $y[1] M\left\{\right.$ vas-Cas9\}ZH-2A $w^{1118}$ females (BDSC_51323) crossed with transgenic dBigH1 gRNA expressing males. Gene replacements were identified by $d s R e d$ expression in male descendants of injected males (in the case of bigH1NSTOP allele) or PCR on genomic DNA using primers specific for mCherry (mCherry Fw: 5'-GTGAAGCTGCGCGGCACC-3') and dBigH1 (dBigH1 rev0: 5'-TGTGGAGAATACCTATGACGATTGCG-3') (in the case of bigH1NULL) and Sanger sequencing of genomic DNA of both mutants, as well.

\section{Determination of embryo hatching rate}

Homozygous mutant and wild type embryos were collected at $25^{\circ} \mathrm{C}$ and hatching rates were determined $36 \mathrm{~h}$ after egg laying by counting hatched and unhatched embryos in three independent replicates of each genotype (50-200 embryos/replicate, 120 on average).

\section{Antibodies}

Rabbit $\alpha \mathrm{dH} 1$ (1:8000 for IF) was kindly provided by Dr. J. Kadonaga and is described in (Bayona-Feliu et al., 2017). Rabbit $\alpha \mathrm{dBigH} 1$ (1:400 for IF; 1:5000 for WB) is described in (Pérez-Montero et al., 2013). $\alpha \mathrm{H3}$ (1:2000 for WB) was commercially available (Cell Signaling 9715S).

\section{Immunofluorescence analysis}

Immunostaining of embryos was performed according to standard methods. In brief, 0-2h embryos were collected in peach juice plates, dechorionated with $50 \%$ bleach for $2 \mathrm{~min}$, fixed for $20 \mathrm{~min}$ in $47 \%$ heptane and 5.8\% formaldehyde, 
and devitellinized in $\mathrm{MeOH}$. The embryos were then permeabilized in PBS, $0.3 \%$ Triton; blocked in PBS, $0.3 \%$ Triton, $2 \% \mathrm{BSA}$; and incubated at $4{ }^{\circ} \mathrm{C}$ overnight with the indicated antibodies. For visualization, slides were mounted in Mowiol (Calbiochem-Novabiochem) containing $0.2 \mathrm{ng} / \mu \mathrm{l}$ DAPI (Sigma) and visualized in a confocal microscope (Leica SPE).

\section{Western blot (WB) analysis}

Total protein extracts were obtained by homogenizing 40 ovaries in SDS-PAGE loading buffer. WB was carried out according to standard methods.

\section{RESULTS}

We generated two null bigH1 CRISPR alleles: bigH1NULL, in which the complete coding sequence of bigH1 was replaced by mCherry, and bigH1 ${ }^{N S T O P}$, in which an early stop was generated into the $\mathrm{N}$-terminal domain of $\mathrm{dBigH} 1$ (Figure 1A) (see Materials and Methods). These two mutations were homozygous viable and showed no significant difference in embryo hatching rate when compared to control $w^{1118}$ wild-type flies (Figure 1B). The null nature of these mutations was confirmed by western blot (WB) and immunofluorescence (IF) analyses that showed no detectable dBigH1 in ovaries and early embryos from homozygous bigH1NULL and bigH1NSTOP flies (Figures 1C and 1D). These results show that lack of bigH1 does not significantly affect embryo viability, which is in agreement with Li et al. (2019).

Next, we analyzed whether, in null bigH1 ${ }^{N U L L}$ and bigH1 ${ }^{\text {NSTOP }}$ early embryos, the lack of $\mathrm{dBigH} 1$ was compensated by the early expression of maternal $\mathrm{dH} 1$ as proposed in Li et al. (2019). Earlier to nuclear cycle (nc) 7, we could not detect $\alpha \mathrm{dH} 1$ immunostaining in control wild-type embryos (Figure 2A). Instead, we observed intense $\alpha \mathrm{dH} 1$ immunostaining in $100 \%$ of homozygous bigH $1^{N U L L}(\mathrm{~N}=$ 40) and bigH1NSTOP $(\mathrm{N}=30)$ embryos before nc 7 (Figures 2C and 2D), confirming the compensatory $\mathrm{dH} 1$ expression described in Li et al. (2019).

Results reported above and in Li et al. (2019) are in contrast with the high lethality observed in homozygous bigH1 $100 /$ big H1 ${ }^{100}$ embryos (Pérez-Montero et al., 2013). This lethality was observed in crosses between heterozygous bigH1 $100 / T M 6 B$ parents, in which the maternal $\mathrm{dBigH} 1$ contribution was reduced 
but not abolished (Pérez-Montero et al., 2013). Thus, we wonder whether the compensatory $\mathrm{dH} 1$ expression was activated in these embryos. For this purpose, we performed IF experiments that failed to detect significant $\alpha \mathrm{dH} 1$ signal in embryos before nc $7(\mathrm{~N}=28)$ (Figure 2B), which is in contrast with the high reactivity detected in homozygous bigH1 ${ }^{\text {NULL }}$ and bigH1NSTOP mutant embryos (Figures $\mathbf{2 C}$ and 2D). These results indicate that reducing the maternal dBigH1 contribution in bigH1 $100 / T M 6 B$ flies is not sufficient to activate compensatory $\mathrm{dH} 1$ expression.

\section{DISCUSSION}

Results presented here confirmed those previously reported in Li et al. (2019), showing that the lack of embryonic dBigH1 linker histone activates the compensatory expression of the somatic $\mathrm{dH} 1$ counterpart in early embryos, at stages in which $\mathrm{dH} 1$ is normally not expressed. As a consequence, embryos lacking $\mathrm{dBigH} 1$ show no detectable developmental defects and are viable. In contrast, embryos coming from heterozygous bigH1100 mothers do not show compensatory $\mathrm{dH} 1$ expression, likely because their maternal $\mathrm{dBig} H 1$ content is sufficiently high. In this regard, the lethality of homozygous bigH1 $100 / \mathrm{bigH} 1^{100}$ embryos might indicate a failure to compensate their reduced maternal $\mathrm{dBigH} 1$ contribution with the expression of somatic $\mathrm{dH} 1$. However, we cannot exclude the possibility that a secondary mutation accumulated in the stock is causing this lethality. Other important questions remain equally unanswered. For instance, it would be interesting to determine whether the lack of any linker histone causes embryo lethality. Finally, the mechanism by which the lack of $\mathrm{dBigH} 1$ activates early expression of maternal $\mathrm{dH} 1$ also needs to be investigated.

\section{ACKNOWLEDGEMENTS}

We are thankful to Dr. J. Kadonaga for the $\alpha \mathrm{dH} 1$ antibodies. This work was supported by grants from the MICINN (PGC2018-094538-B-100) and the "Generalitat de Catalunya" (SGR2017-475) to FA, and the Hungarian National Scientific Research Fund (OTKA-116372) to IB and LH. 


\section{REFERENCES}

Bayona-Feliu, A., Casas-Lamesa, A., Carbonell, A., Climent-Cantó, P., Tatarski, M., Pérez-Montero, S., Azorín, F., and Bernués, J. (2016). Histone H1: Lessons from Drosophila. Biochim Biophys Acta 1859, 526-532.

Bayona-Feliu, A., Casas-Lamesa, A., Reina, O., Bernués, J., and Azorín, F. (2017). Linker histone $\mathrm{H} 1$ prevents R-loop accumulation and genome instability in heterochromatin. Nat Commun 8, 283.

Bischof, J., Maeda, R.K., Hediger, M., Karch, F., and Basler, K. (2007). An optimized transgenesis system for Drosophila using germ-line-specific phiC31 integrases. Proc Natl Acad Sci USA 104, 3312-3317.

Carbonell, A., Pérez-Montero, S., Climent-Cantó, P., Reina, O., and Azorín, F. (2017). The germline linker histone $\mathrm{dBigH} 1$ and the translational regulator Bam form a repressor loop essential for male germ stem cell differentiation. Cell Rep 21, 3178-3189.

Jeong, J.-Y., Yim, H.-S., Ryu, J.-Y., Lee, H.S., Lee, J.-H., Seen, D.-S., and Kang, S.G. (2012). One-step sequence- and ligation-independent cloning as a rapid and versatile cloning method for functional genomics studies. Appl Environ Microbiol 78, 5440-5443.

Li, K.K., Han, D., Chen, F., Li, R., Zhou, B.-H., Bai, Y., Yuan, K., and Rong, Y.S. (2019). Compensatory replacement of the BigH1 variant histone by canonical H1 supports normal embryonic development in Drosophila. bioRxiv doi: http://dx.doi.org/10.1101/789735.

Pérez-Montero, S., Carbonell, A., and Azorín, F. (2016). Germline-specific H1 variants: the "sexy" linker histones. Chromosoma 125, 1-13.

Pérez-Montero, S., Carbonell, A., Morán, T., Vaquero, A., and Azorín, F. (2013). The embryonic linker histone $\mathrm{H} 1$ variant of Drosophila, $\mathrm{dBigH} 1$, regulates zygotic genome activation. Dev Cell 26, 578-590.

Port, F., Chen, H.-M., Lee, T., and Bullock, S.L. (2014). Optimized CRISPR/Cas tools for efficient germline and somatic genome engineering in Drosophila. Proc Natl Acad Sci USA 111, E2967-2976. 


\section{FIGURE LEGENDS}

Figure 1. Generation of null bigH1 CRISPR alleles. A) Schematic representation of the bigH1NULL and bigH1NSTOP alleles. B) The rate of embryo hatching is presented for the indicated genotypes. C) Western-blot analysis with $\alpha \mathrm{dBigH} 1$ antibodies of increasing amounts (lanes 1-3) of total extract prepared from ovaries of females of the indicated genotypes. $\alpha \mathrm{H} 3$ was used for loading control. D) Immunofluorescence staining with $\alpha \mathrm{dBigH} 1$ antibodies (in green) of early Drosophila embryos ( $\mathrm{nc}=2$ ) of the indicated genotypes. DNA was stained with DAPI (in red).

Figure 2. Compensatory dH1 expression. A) Immunofluorescence staining with $\alpha \mathrm{dH} 1$ antibodies (in green) of wild-type Drosophila embryos at the cellular blastoderm stage (left) and before nc 7 (right). DNA was stained with DAPI (in red). Insets show enlarged images. B) As in A but for embryos from bigH1 $100 / T M 6 B$ parents. C) As in A but for embryos from homozygous bigH1 ${ }^{\text {NSTOP }}$ parents. D) As in A but for embryos from homozygous bigH1 NULL parents. 
A bigH1NSTOP

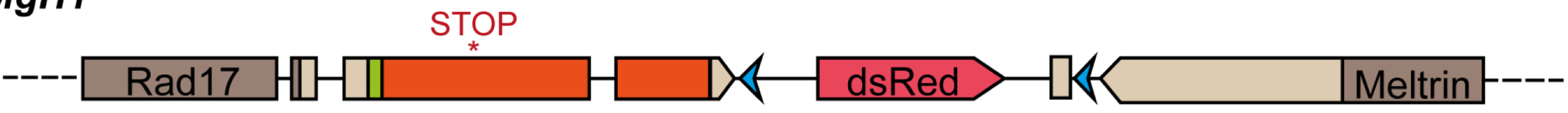

bigH1 ${ }^{\text {NULL }}$

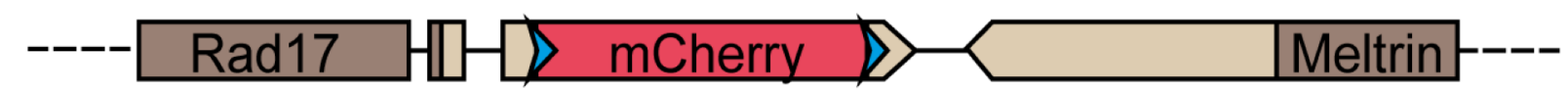

$\square$ Neighbouring genes $\quad \square$ UTRs $\quad \square$ 3x FLAG-tag $\square$ BigH1 $\quad$ LoxP site

B

\section{B Embryo hatching rate}

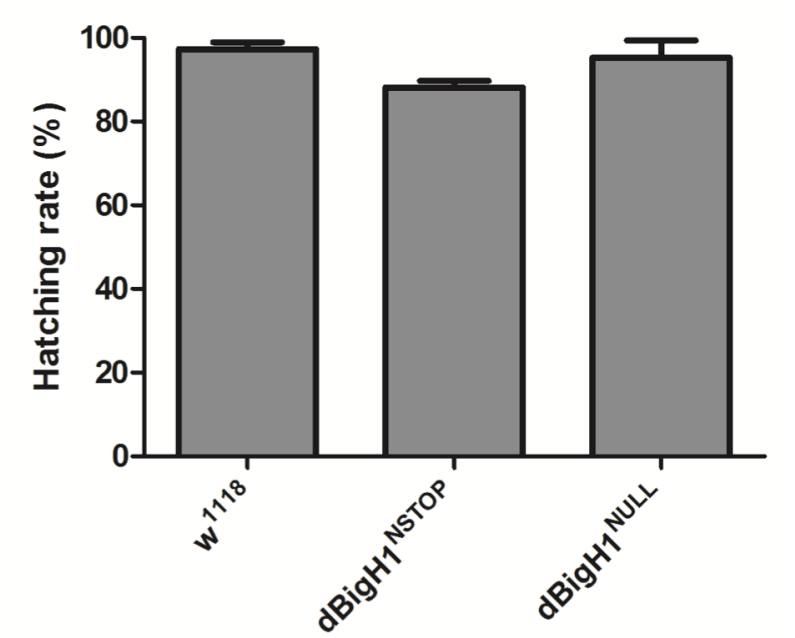

C

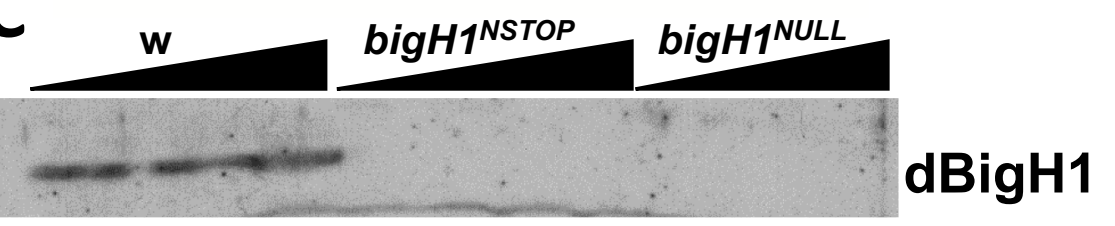

1
D

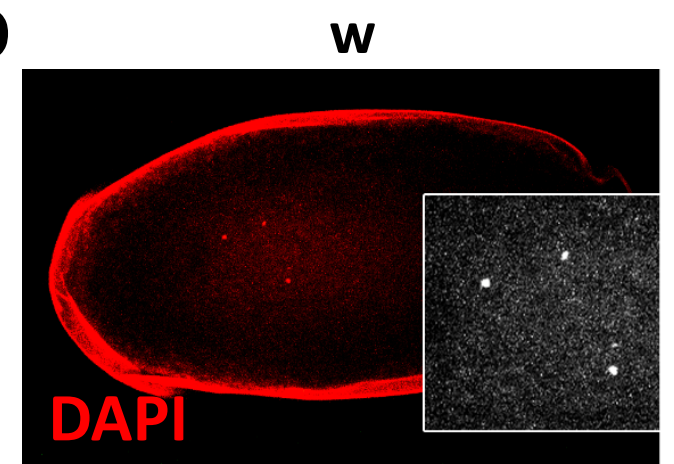

dBigH1

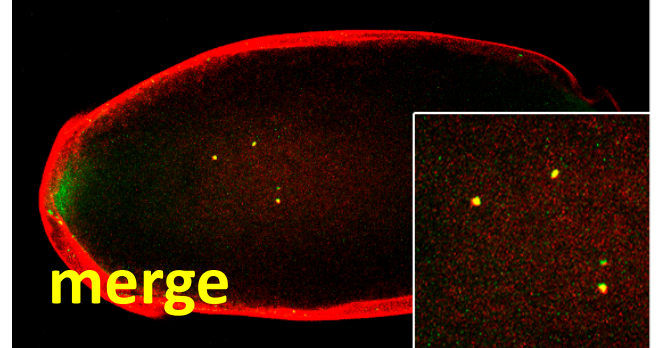

dBigH1 ${ }^{\text {NSTOP }}$

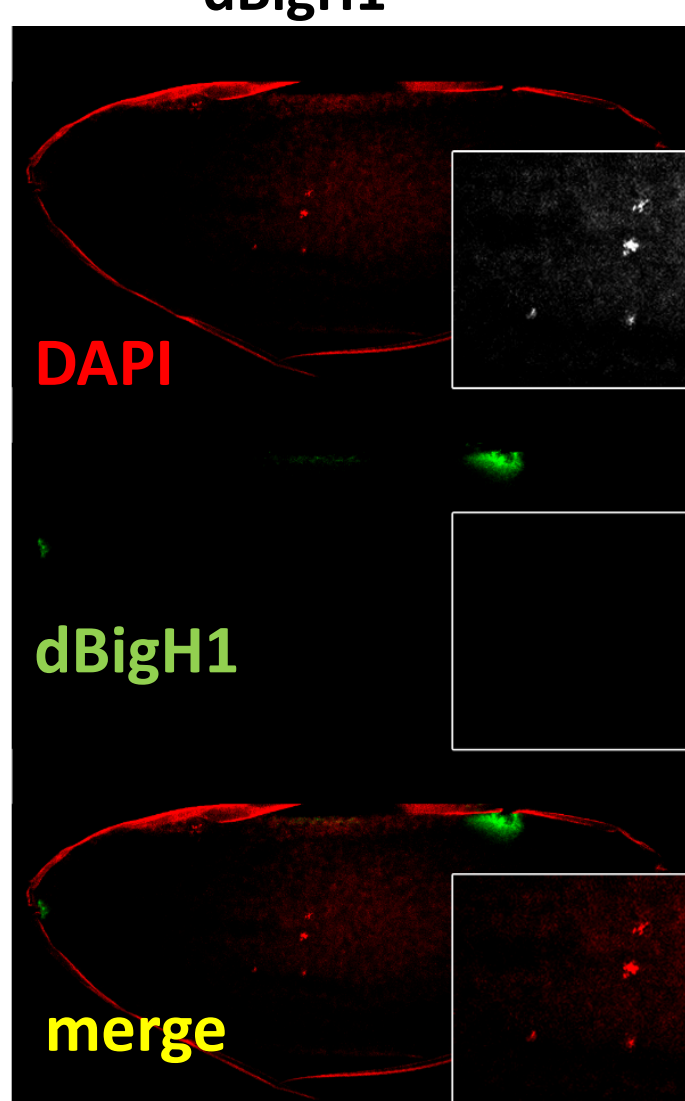

dBigH1 $^{\text {NULL }}$

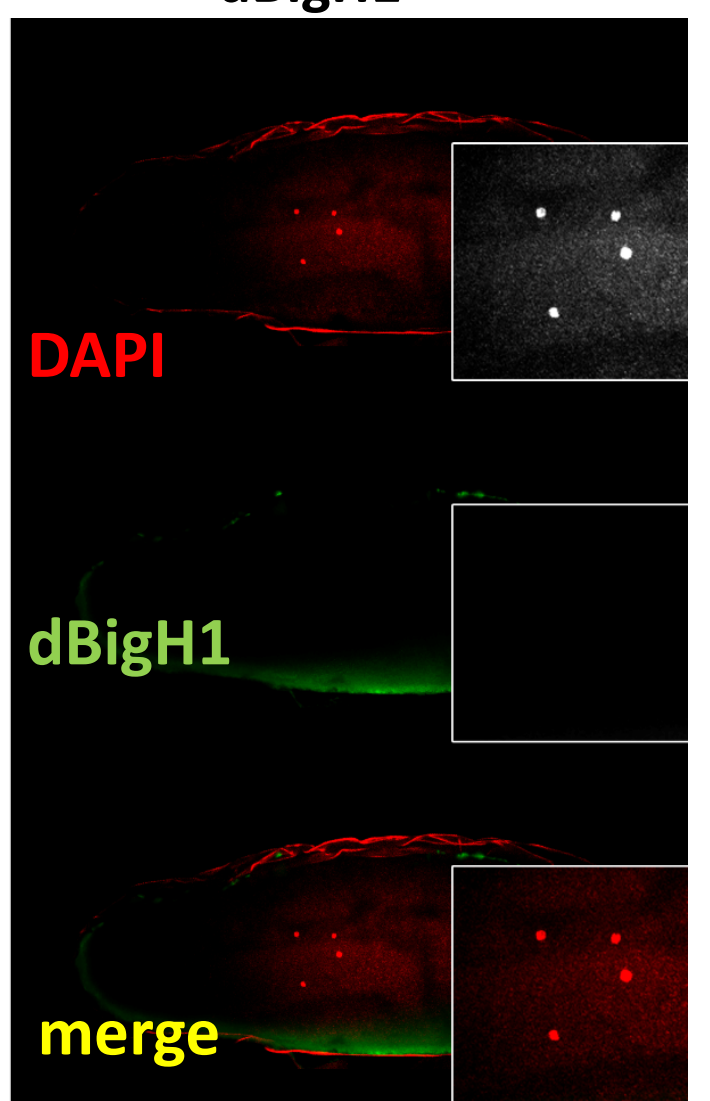



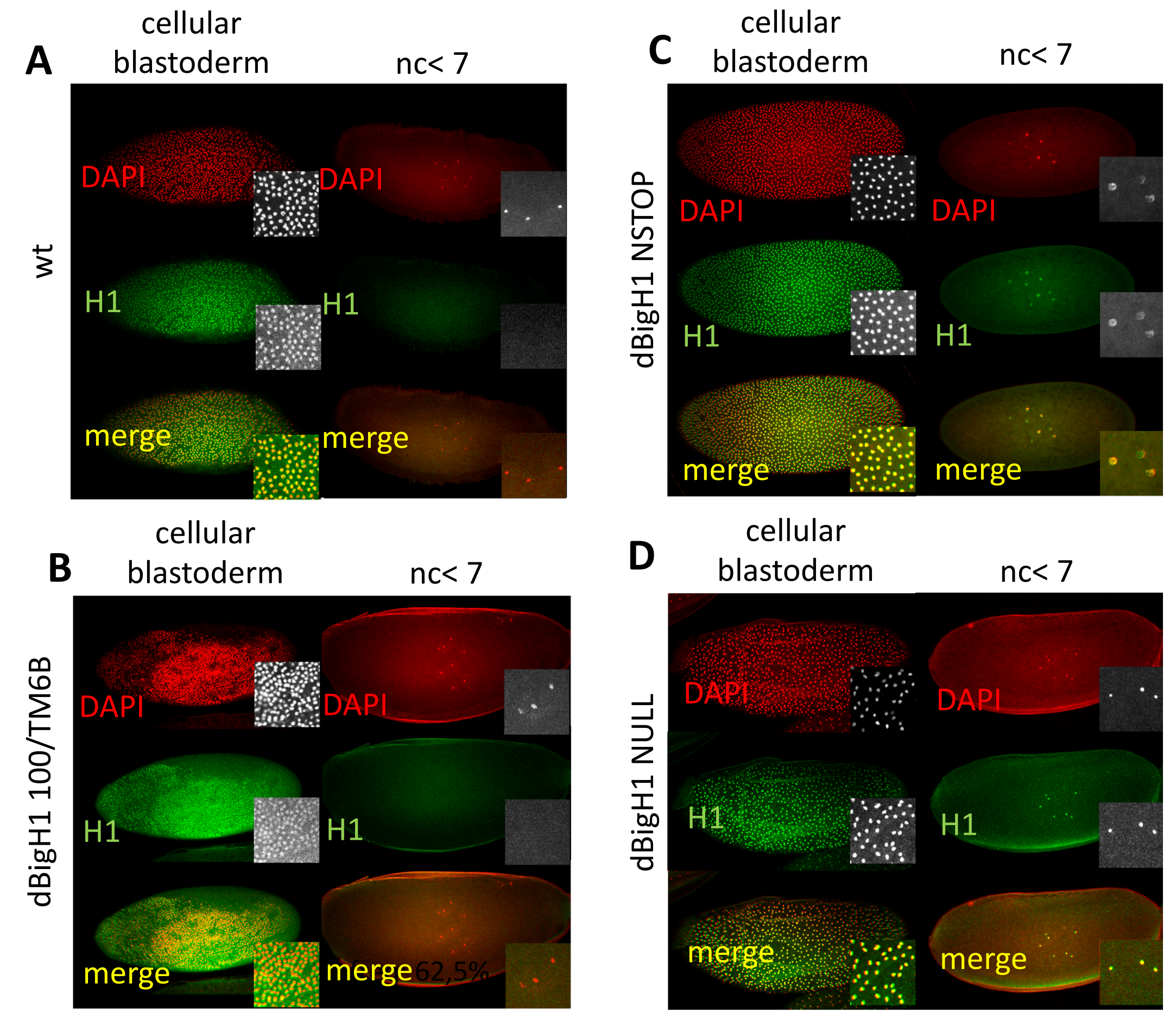